\title{
Trabajo colaborativo y desempeño profesional: un caso práctico en la clase de accesibilidad audiovisual
}

\section{(Collaborative work and job performance: a case study in the audiovisual accessibility classroom)}

\author{
Beatriz Cerezo Merchán \\ IRENE DE HIGES ANDINO \\ Universitat Jaume I
}

Fecha de recepción: 26 de junio de 2013

Fecha de aceptación: 15 de julio de 2013

Resumen: Dentro del marco del Espacio Europeo de Educación Superior (EEES), que promueve enfoques cognitivo-constructivistas, el estudiante se convierte en protagonista de su proceso de aprendizaje, basado en la adquisición de competencias. Dentro de estos enfoques, el aprendizaje colaborativo y el acercamiento a la práctica profesional resultan instrumentos clave para que los alumnos adquieran las competencias deseadas. Siguiendo estas premisas, las autoras de este artículo desarrollaron un proyecto de innovación educativa aplicado a la formación de traductores en el ámbito de la accesibilidad audiovisual (audiodescripción para personas con discapacidad visual y subtitulado para personas sordas y personas con discapacidad auditiva). En este artículo se presentan las acciones desarrolladas dentro del proyecto, así como reflexiones sobre los resultados obtenidos y propuestas de acciones futuras en el aula.

Palabras clave: Formación de traductores, Competencias, Trabajo colaborativo, Práctica profesional, Audiodescripción, Subtitulación para sordos

Abstract: Within the European Higher Education Area (EHEA) cognitive constructivist approaches are promoted, which means that students are transformed into the protagonists of their learning process, which is based on the acquisition of competences. Within this scheme, collaborative learning and the approach to professional practice are key tools for students to acquire the desired competences. Following these ideas, the authors of this article developed an educational innovation project aimed at the training of translators in the field of audiovisual accessibility (audio description for the 
blind and partially sighted and subtitling for the deaf and hard-of-hearing). In this article we present the activities developed during the project together with some reflections on the results. Finally we suggest new exercises to be implemented in class in the future.

Key words: Translator training, Skills, Collaborative work, Professional practice, Audio-description, Subtitling for the deaf.

\section{INTRODUCCIÓN}

La didáctica de la Traducción es una disciplina relativamente joven, que empezó a desarrollarse a mediados del siglo XX. En sus inicios, la didáctica de la Traducción se basaba en modelos de enseñanza puramente transmisionistas, pero a partir de la década de los 80 empezaron a surgir enfoques innovadores, en línea con las bases de modelos de enseñanza transaccionales y transformacionales, centrados en el aprendizaje colaborativo y en el control del alumno sobre su propio proceso de aprendizaje (González Davies, 2004). En el periodo actual de armonización de los sistemas educativos europeos para la consolidación del EEES también se apuesta por modelos didácticos cognitivo-constructivistas, que consideran al estudiante como el protagonista de su propio aprendizaje y apuestan por una estrecha relación entre formación universitaria y mercado laboral. En este contexto, el papel de las competencias, tanto genéricas como específicas, ocupa el centro del discurso pedagógico, ya que, unido a otros conceptos clave como los objetivos y resultados de aprendizaje o los perfiles profesionales y la empleabilidad, es imprescindible para impulsar las reformas educativas y establecer un vínculo directo entre la formación, la sociedad y el mercado. Teniendo en cuenta este nuevo paradigma educativo, parece lógico que, como veremos con mayor detalle a continuación, tengan especial importancia las competencias interpersonales, que capacitan al profesional para trabajar en equipo, comunicarse con expertos de otras áreas, apreciar la diversidad y multiculturalidad, etc.

En el campo de la formación de traductores e intérpretes los modelos competenciales vienen empleándose casi desde los inicios de nuestra disciplina con el objetivo de configurar el perfil del traductor y el intérprete y separarlo de la figura del profesional de las lenguas o la comunicación (Calvo y Morón, 2008). Desde el punto de vista de la propia evolución de la disciplina, existen numerosos trabajos sobre el concepto de competencia traductora en la formación de traductores, que muestran diferentes enfoques y aplicaciones, desde el modelo más "sencillo", el modelo minimalista de Pym (1991), hasta los más complejos, compuestos por 
distintas competencias y subcompetencias que integran la competencia traductora. Los dos modelos más completos que existen hasta la fecha, y que recogen la mayor parte de las propuestas de autores anteriores, reestructuradas y ampliadas, son el modelo de PACTE $(2001,2003)$ y el de Kelly $(2002,2005)$.

En nuestro artículo nos centraremos en el modelo de competencia traductora propuesto por Kelly $(2002,2005)$, ya que en él encontramos aspectos profesionales que no están presentes en otros modelos. Kelly (2002: 14) define la competencia traductora como una "macrocompetencia" que constituye el conjunto de capacidades, destrezas, conocimientos e incluso actitudes que reúnen los traductores profesionales y que intervienen en la traducción como actividad experta. Según este modelo, la competencia traductora puede dividirse en siete subcompetencias, que podemos observar en la siguiente figura:

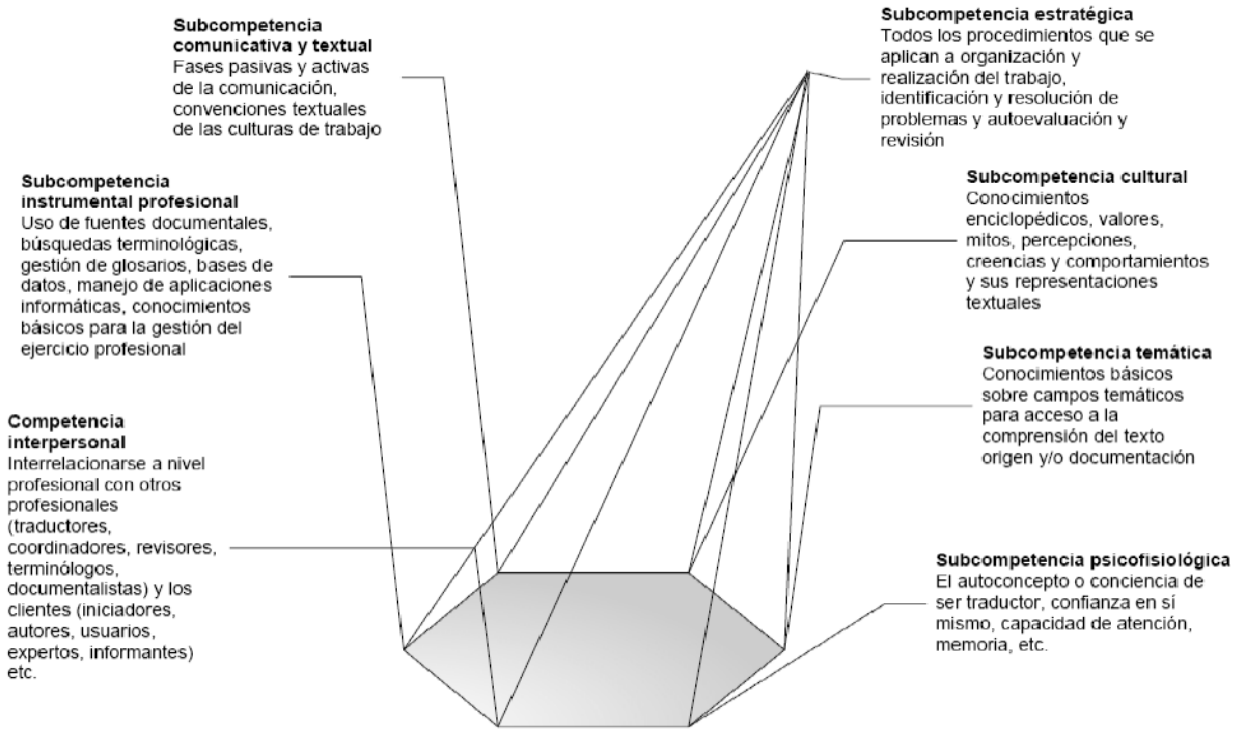

Modelo de competencia traductora de Kelly (2002, 2005)

Como vemos, dentro de la competencia interpersonal encontramos el trabajo en equipo como elemento imprescindible para que el traductor conozca las dinámicas de trabajo real de la traducción, sea capaz de trabajar codo con codo con otros profesionales y con los clientes, y pueda, por tanto, integrarse en el mercado laboral satisfactoriamente. En línea con 
esta propuesta se encuentra el Proyecto Tuning (González y Wagenaar, 2003), un plan de convergencia elaborado por y para las universidades en respuesta al Proceso de Bolonia, que se centra en la búsqueda y desarrollo de competencias como medio para alcanzar perfiles profesionales y académicos. En particular, este proyecto se centra en dos tipos de competencias, las genéricas y las específicas. Como pilar fundamental de las competencias genéricas, encontramos las competencias interpersonales, que son las siguientes: habilidades de crítica y autocrítica, trabajo en equipo, capacidad para trabajar en equipos multidisciplinares, habilidades para trabajar con expertos de otras áreas, apreciación por la diversidad y multiculturalidad, habilidades para trabajar en contextos internacionales y compromiso ético. También en esta misma línea, encontramos la propuesta del Libro Blanco de Grado en Traducción e Interpretación (Muñoz Raya, 2003), en la que se determina que el trabajo en equipo figura como una de las diez competencias, en este caso específicas, más importantes para el desempeño profesional de traductores e intérpretes.

\section{EL APRENDIZAJE COLABORATIVO EN LOS ESTUDIOS DE TRADUCCIÓNE INTERPRETACIÓN}

Dentro de los nuevos enfoques pedagógicos del ámbito concreto de la traducción, en línea con la filosofía del EEES, el diseño curricular se realiza a partir de las necesidades reales de la sociedad y del mercado, que se definen en forma de competencias y perfiles profesionales. De acuerdo con este enfoque, Kelly (2005: 28) afirma lo siguiente: "If we are to describe our overall aims in terms of what professionals are required to do and to know, we need to be able to systematize the common denominator of what translators actually do in their daily work". En esta misma línea, en el apartado anterior hemos comprobado que propuestas actuales de competencias traductoras basadas en la práctica real profesional de la traducción sitúan el trabajo colaborativo dentro de las principales competencias específicas de los traductores, ya este es cada vez más importante en la labor de los traductores de hoy en día (Huertas Barros, 2011).

Para hacer frente a estas necesidades, en los últimos años han surgido diferentes propuestas metodológicas colaborativas en el ámbito de la enseñanza de la traducción. Su máximo exponente es Kiraly (2000), quien propone una metodología basada en la realización de lo que él denomina Authentic Collaborative Translation Projects, verdaderos encargos de traducción realizados de forma colaborativa por equipos de estudiantes junto con el profesor, que actúa como facilitador y guía de los proyectos. En palabras del autor: "[...] the workshop can be a venue where 
authentic practice can occur among peers learning with (not from) a facilitator to construct their own understandings of social phenomena" (Kiraly, 2000: 63). En su propuesta, Kiraly destaca la importancia de que los encargos de traducción se realicen realmente de forma colaborativa, es decir, de que se trabaje en equipos en lugar de en grupos, ya que esta forma de trabajo garantiza que los estudiantes no se limiten a dividir entre ellos la tarea a realizar, sino que requiere que realicen la tarea y construyan significado conjuntamente, y desarrollen juntos conocimientos culturales y profesionales.

En la actualidad, es cada vez más frecuente el empleo de propuestas como las de Kiraly en la clase de traducción. Para su aplicación, el profesorado recurre a la realización de encargos de traducción reales o simulados, en los que el estudiantado trabaja, con frecuencia, desarrollando diferentes roles, tales como la gestión del proyecto, la gestión documental y terminológica, la traducción, la revisión, etc.

Teniendo en cuenta la importancia del trabajo en equipo en el sector de la accesibilidad audiovisual (que abordaremos en el siguiente apartado) y la necesidad de trabajar las competencias interpersonales en la formación de traductores, las autoras de este artículo decidieron poner en práctica metodologías de trabajo colaborativo en la clase de subtitulación para sordos y audiodescripción para ciegos. Sin embargo, antes de pasar a describir este proyecto de innovación docente, y para justificar plenamente las acciones desarrolladas, consideramos necesario presentar, en el siguiente apartado, las dos modalidades de traducción audiovisual objeto de nuestro proyecto, así como cuestiones profesionales de estas, como son el perfil de los profesionales que se dedican a ellas y las formas habituales de trabajo en este sector.

\section{EL MERCADO LABORAL DE LA TRADUCCIÓN AUDIOVISUAL Y LA ACCESIBILIDAD}

Con la Ley $7 / 2010$, de 31 de marzo, General de la Comunicación Audiovisual, el Estado español transpone la Directiva 2007/65/CE de Servicios de Comunicación Audiovisual del Parlamento Europeo y del Consejo de 11 de diciembre del 2007. Dado que hoy en día los materiales audiovisuales son la base de nuestra comunicación, esta directiva -y su consecuente transposición a la legislación española- pretende suplir las carencias de aquellos colectivos que tienen mermados los sentidos del oído y la vista. Las técnicas empleadas son el subtitulado para personas sordas y personas con discapacidad auditiva y la audiodescripción para personas con problemas visuales, que definimos a continuación.

El subtitulado suele definirse como el medio de apoyo que presenta un "conjunto de texto y gráficos que muestran en pantalla los discursos 
orales, la información contextual y los efectos sonoros que se producen en cualquier obra audiovisual" (AENOR, 2012: 7). El aspecto que diferencia el subtitulado para sordos de la subtitulación convencional es el público al que se dirige: personas que tienen problemas de oído (sordera profunda o pérdida de audición). Por consiguiente, los subtítulos se convierten en voces que se leen al poner por escrito los diálogos y los efectos sonoros, así como las emociones que se perciben por el tono y la modulación de la voz (Neves, 2007). La audiodescripción se describe como:

\begin{abstract}
Un servicio de apoyo a la comunicación que consiste en el conjunto de técnicas y habilidades aplicadas, con objeto de compensar la carencia de captación de la parte visual contenida en cualquier tipo de mensaje, suministrando una adecuada información sonora que la traduce o explica, de manera que [...] [se] perciba dicho mensaje como un todo armónico y de la forma más parecida posible a como lo percibe una persona que ve. (AENOR 2005: 4)
\end{abstract}

A modo de resumen, se fundamenta en narrar la acción, el lenguaje corporal, las expresiones faciales, el escenario y el vestuario sin interferir en los diálogos ni los efectos sonoros o musicales importantes para la obra audiovisual (Benecke, 2004). Al contrario que el subtitulado, se trata de verbalizar lo visual (Snyder, 2008).

La Ley General de la Comunicación Audiovisual ha impulsado el crecimiento del mercado profesional de la accesibilidad desde su aprobación en 2010 , porque determina un aumento progresivo del volumen de emisiones accesibles. Para finales del año 2013, los canales de televisión públicos deberán haber alcanzado las siguientes cifras de emisiones accesibles a la semana: 10 horas de lengua de signos, 10 horas de audiodescripción y el $90 \%$ de las emisiones con subtitulación para sordos. Respecto a los canales televisivos en abierto y cobertura estatal o autonómica, el volumen de accesibilidad se reduce a un $75 \%$ de las emisiones con subtitulación para sordos, dos horas de lengua de signos y dos horas de audiodescripción a la semana. Según el informe publicado por la Comisión del Mercado de las Telecomunicaciones en 2011, en casi todos los canales de TDT ha aumentado el número de horas de subtitulación en comparación con 2009. En el caso de la audiodescripción, observamos que en 2011 su aplicación aún era bastante deficiente (no disponemos de datos estadísticos más recientes). En cualquier caso, en este trabajo, no nos detendremos a evaluar si se cumple la Ley General de la Comunicación Audiovisual, ya que nuestro único objetivo es presentar una propuesta de formación de profesionales en el ámbito de la accesibilidad centrándonos en la subtitulación y la audiodescripción. 
Para los ajenos a este campo, puede resultar extraño que se considere que la accesibilidad forma parte de los Estudios de Traducción y, en concreto, de la traducción audiovisual. Se entiende que los procesos para hacer accesibles los productos audiovisuales no son un ejemplo de traducción interlingüística —probablemente la acepción más habitual cuando se piensa en traducción-, sino que hacen referencia a las otras clases de traducción propuestas por Jakobson (1959). Por un lado, la subtitulación para sordos es un ejemplo de traducción intralingüística, es decir, se crean los subtítulos en la misma lengua en la que se oye el producto audiovisual. Cuando se subtitulan obras audiovisuales extranjeras, se parte de la versión doblada. Esto se debe a que en el proceso de subtitulado para sordos se intenta acercar los subtítulos lo máximo posible a la pista auditiva, porque las personas que tienen restos de audición escuchan habitualmente el audio y emplean el subtitulado como apoyo. Por otro lado, la audiodescripción se considera traducción intersemiótica, ya que se produce un trasvase de la información contenida en el canal visual al canal auditivo. Como señala Benecke (2004), narrar lo que ocurre en pantalla para una persona que no ve es una práctica muy antigua, pero que surge como actividad profesional durante el siglo XX con el propósito de dar autonomía a este colectivo.

En el ámbito español, los servicios de subtitulación para sordos y audiodescripción cuentan con una normativa que establece los requisitos básicos que deberían cumplirse para ofrecer un producto de calidad: la norma UNE 153020 (2005) para la audiodescripción para personas con discapacidad visual y la norma UNE 153010 (2012) para el subtitulado para personas sordas y personas con discapacidad auditiva. Sin embargo, no queda claro quién debería acometer la formación de los profesionales de la accesibilidad. Hoy en día se ofrecen cursos de subtitulado y audiodescripción en empresas del sector y centros de formación continua (Badia y Matamala, 2007). También las universidades ofrecen formación en este campo (principalmente de posgrado).

La Universitat Jaume I, en la que las autoras desarrollamos nuestra labor docente e investigadora, fue pionera en la formación en traducción audiovisual en los años 90 al incorporar un itinerario específico de esta modalidad de traducción en la extinta Licenciatura en Traducción e Interpretación. También fue precursora en la formación en accesibilidad al introducirla en los estudios de licenciatura, pues en 2007 se actualizó el temario de la asignatura Traducción Audiovisual III para dar cabida a este campo (Marzà, 2010). Hoy en día, en el Grado de Traducción e Interpretación de la Universitat Jaume I, el subtitulado para sordos y la audiodescripción forman parte de los contenidos de la asignatura obligatoria 
de tercer curso Traducción Audiovisual (6 créditos ECTS). Tras dicha introducción, los alumnos pueden optar por profundizar en este ámbito al matricularse en cuarto curso en la asignatura optativa Traducción Audiovisual y Accesibilidad (4,5 créditos ECTS). La implantación de esta asignatura en el curso académico 2012/2013 es el marco en el que se sitúa la propuesta docente que presentamos en este artículo.

Tomamos como punto de partida los conocimientos y las habilidades recomendables para realizar una audiodescripción y un subtitulado para sordos de calidad que se ven reflejados en la práctica diaria de la profesión. Partimos de las propuestas de Álvarez Doblas e Hidalgo Valdés (2006), Díaz Cintas (2007), Matamala y Orero (2007), Neves (2008) y Snyder (2008). Como Díaz Cintas (2007), presentamos las competencias divididas en cuatro categorías: lingüísticas, temáticas, tecnológicas y generales.

Competencias lingüísticas:

- Dominio de la lengua materna, en todas sus dimensiones: fonética, léxico, ortografía, gramática y sintaxis.

- Capacidad de síntesis.

- Conocimiento de los conceptos de redundancia, relevancia, adecuación cohesión y coherencia.

- Rigor para revisar la calidad del propio trabajo o del trabajo de otros.

- Buena dicción en el caso de la audiodescripción'

Competencias temáticas o de contenido:

- Cultura de la accesibilidad y el diseño para todos.

- Acercamiento al perfil y las necesidades de las personas con problemas de visión u oído.

- Introducción en la historia y la evolución de la audiodescripción y el subtitulado para sordos.

- Conocimiento del mercado laboral y la legislación sobre AD y SPS.

- Cinematografía.

- Reconocimiento de la multimodalidad de los textos audiovisuales y los códigos que los conforman.

- Capacidad de análisis de textos audiovisuales.

- Peculiaridades de los diferentes géneros audiovisuales.

\footnotetext{
${ }^{1}$ En España, los actores de doblaje realizan la locución, a excepción de la audiodescripción en directo en la que el propio audiodescriptor lee su trabajo.
} 
Competencias tecnológicas y aplicadas:

- Manejo avanzado de herramientas informáticas, en especial en el subtitulado para sordos.

- Conocimientos avanzados de documentación.

Personales y generales:

- Conciencia del papel intermediario de la labor del audiodescriptor y el subtitulador para sordos.

- Capacidad de observación, en especial en la audiodescripción, pues se describe lo que se ve, tal y como enuncia Snyder (2008: 195): "What You See Is What You Say".

- Capacidad de organización y planificación.

- Habilidades interpersonales que faciliten la integración y la dirección de equipos de trabajo.

Con la perspectiva de trabajo colaborativo que presentamos en este artículo, es importante conocer cómo se trabaja en el ámbito de la accesibilidad. La norma UNE 153020 (2005) indica que en España la audiodescripción debería ser fruto del trabajo de un audiodescriptor y de un revisor. Así se trabaja, por ejemplo, en Televisió de Catalunya (Matamala y Orero, 2008). En nuestra propuesta docente, adoptamos el método empleado en la televisión regional alemana Bayerischer Rundfunk, donde desde 1997 se emiten programas audiodescritos con frecuencia. En esta televisión la audiodescripción es el producto del trabajo conjunto de dos audiodescriptores, que, además, cuentan con el apoyo y la revisión de una tercera persona con problemas de visión. Matamala y Orero (2007) ya aplican este método de trabajo colaborativo en el aula de audiodescripción.

En el ámbito de la subtitulación para sordos, el trabajo en equipo era habitual en Televisió de Catalunya en el subtitulado en directo, pues se diseñó un sistema de semáforos en los que cinco personas trabajan al mismo tiempo encargándose cada una de ellas de un fragmento. La necesidad de concentración y compenetración era en este caso imprescindible. En nuestra propuesta docente, reproducimos la realidad profesional que compete también al proceso de subtitulación convencional.

Sánchez (2004) describe varias fases del trabajo de subtitulación, que pueden ser llevadas a cabo por diferentes personas y, por tanto, son tareas que pueden formar parte de una cadena de trabajo en equipo. Las fases son las siguientes:

- Pretraducción: Traducción literal del texto audiovisual sin crear subtítulos. 
- Adaptación: Aplicación del formato de los subtítulos a un texto ya traducido.

- Pautado: Creación de los subtítulos con sus correspondientes tiempos de entrada y salida.

- Traducción: Trasvase lingüístico de un texto original a la lengua meta. En el caso del subtitulado para sordos, el trasvase es, en general, intralingüístico.

- Revisión: Por un lado, lectura del archivo de subtítulos sin tener acceso a la imagen, para detectar erratas y frases sin sentido. Por otro, realización de un visionado con simulación (se reproduce cómo se verán los subtítulos en el producto final).

3. EXPERIENCIA DE TRABAJO POR EQUIPOS Y ACERCAMIENTO A LA PRÁCTICA PROFESIONAL ENEL AULA DE ACCESIBILIDAD AUDIOVISUAL

Como comentábamos anteriormente, la importancia de las competencias interprofesionales y del trabajo en equipo en la traducción en general, y en el sector de la accesibilidad audiovisual en particular, fue lo nos llevó a plantear la puesta en práctica de metodologías de trabajo colaborativo en la clase de subtitulación para sordos y audiodescripción para ciegos. Concretamente, diseñamos una acción de innovación docente, integrada en el Programa de formación de profesorado novel de la Universitat Jaume I, denominada "Acercamiento de la práctica profesional al aula en la asignatura Traducción audiovisual y accesibilidad", que realizamos durante el curso 2012/2013.

En este proyecto, como ya hemos mencionado, intentamos aproximar a los estudiantes a las dinámicas de trabajo reales del ámbito de la subtitulación para sordos y la audiodescripción para ciegos. De esta manera, prestamos especial importancia a trabajar en encargos colaborativos de traducción simulados en los que los alumnos trabajaron con roles asignados y con el profesor como guía, pero también a presentar cuestiones prácticas del mercado laboral de la traducción audiovisual y la accesibilidad de la mano de profesionales, a hacer reflexionar al estudiante sobre su proceso de aprendizaje, etc. A continuación presentamos, en primer lugar, los objetivos específicos que se persiguieron con la aplicación de metodologías de trabajo colaborativo en la clase de accesibilidad audiovisual, y a continuación, las dinámicas concretas que desarrollamos.

\subsection{Objetivos}

Al proponer esta experiencia de trabajo colaborativo y acercamiento del mundo profesional, nos planteamos los siguientes objetivos específicos: 
- Hacer que los estudiantes desarrollen habilidades de resolución de problemas de manera autónoma y en equipo. Con los distintos encargos de traducción que se plantean en el aula, el alumnado se ve en la tesitura de entregar un producto de calidad teniendo en cuenta las restricciones específicas del texto audiovisual y el ambiente de trabajo reproducido, de modo que aprende a resolver los problemas que surjan en colaboración con otros compañeros o por sí mismo.

- Trabajar con los estudiantes en el manejo de fuentes de documentación sobre lengua, cultura y cine que están al alcance del estudiante. La subcompetencia instrumental profesional (Kelly, 2002, 2005) es sin lugar a dudas clave en la formación de traductores. En el aula de la accesibilidad, es imprescindible adoptar un enfoque interdisciplinario mediante el cual el alumnado disponga de recursos para documentarse no solo sobre lengua y cultura, sino también sobre cinematografía.

- Dar a conocer los protocolos de trabajo de la industria de la accesibilidad en España. Como en otros ámbitos de la traducción, los protocolos de trabajo dependen en muchas ocasiones de las exigencias, costumbres y normas internas de cada cliente o empresa. No obstante, con las tareas de clase y la presencia de profesionales en el aula, el alumnado tendrá la disponibilidad de aprender cómo se trabaja en diferentes empresas del sector.

- Trabajar en la aplicación de las normas profesionales de la subtitulación para sordos y la audiodescripción para ciegos en España. La norma UNE 153010 (2012) y la norma UNE 153020 (2005) conforman el marco metodológico a partir del cual se prepara una propuesta docente dirigida a establecer un vínculo directo entre la formación, la sociedad y el mercado.

\subsection{Actividades realizadas}

Como paso previo a realizar los trabajos colaborativos de ambos bloques de la asignatura, en anteriores sesiones se trabajó en el visionado y posterior debate de vídeos sobre ceguera, sordera y temas relacionados con la audiodescripción y la subtitulación para sordos. También se presentaron cuestiones generales y profesionales de ambas modalidades de traducción y se analizaron las convenciones de la audiodescripción (Norma UNE 153020: 2005) y de la subtitulado (Norma UNE 153010: 2012). Respecto a esta última modalidad, se compararon las convenciones de la SPS con la subtitulación convencional.

Asimismo, se realizaron ejercicios de análisis de vídeos audiodescritos y subtitulados para sordos grabados de la televisión y fragmentos audiodescritos extraídos de DVD. El alumnado tuvo la posibilidad de entregar ejercicios voluntarios e individuales de fragmentos 
de programas (principalmente dibujos y películas) con diferentes problemas de traducción. En audiodescripción, se trabajó la presentación de los personajes y la descripción de los créditos, los diálogos multilingües, las escenas de violencia, las comedias, etc, En SPS, algunos fragmentos eran muy apropiados para trabajar las emociones, otros para trabajar la música, otros para trabajar elementos sonoros y onomatopeyas, etc. Así, cuando llegamos a las actividades colaborativas, los alumnos ya conocían todo el proceso de la AD y la SPS y sus convenciones, sabían utilizar programas de edición de audio y subtítulos y habían trabajado las distintas dificultades que esta modalidad de traducción entraña.

\subsubsection{Encargos en equipo tutelados}

A lo largo del curso académico, los alumnos trabajan en equipos de tres personas para realizar tareas basadas en el trabajo colaborativo con roles asignados.

\section{Encargos de audiodescripción}

En audiodescripción se replica el proceso de trabajo de la televisión alemana Bayerischer Rundfunk (Benecke, 2004), que Matamala y Orero (2007) aplican al aula de audiodescripción en España. Se les plantean dos encargos "reales" que deben acometer a tres bandas teniendo en cuenta que uno de los miembros del grupo ejerce de persona sin visión. Mientras que en la televisión alemana la persona ciega colabora como revisor; en esta actividad la persona que no ve el vídeo trabaja desde el principio con sus compañeros.

El encargo consiste en la audiodescripción y locución de un fragmento de vídeo de unos cuatro minutos. Durante el curso académico 2012/2013, nos detenemos en la audiodescripción de un fragmento de un filme infantil de animación y un documental, cuya audiodescripción presenta unos rasgos característicos (véase ITC 2000; Palomo 2008; RNIB 2009; Cámara y Espasa 2011). Aunque en España no es muy habitual que los audiodescriptores sean las personas que se encargan de la locución, el encargo incluye esta fase de postproducción que permite que el alumnado sea aún más consciente si cabe del ajuste. Además, la norma UNE recomienda que en obras infantiles "el locutor o locutora utilice una entonación adecuada para niños, pudiendo ser algo más expresiva" (AENOR, 2005: 9). En nuestro caso, se trabaja en el aula el funcionamiento del programa Windows Movie Maker, que permite añadir la narración al fragmento de vídeo.

Describimos, a continuación, los materiales de que disponen los alumnos y el proceso de trabajo. Para esta tarea el alumnado cuenta con un 
archivo de vídeo con el fragmento que debe audiodescribir y un archivo de audio en el que solo se escucha la pista sonora de dicho fragmento. Disponen de quince días para realizar el encargo, que se comienza en el aula, pero que principalmente tiene lugar fuera de ella. Aproximadamente a los diez días tiene lugar una tutoría de carácter obligatorio con la profesora para comentar el proceso de trabajo y resolver dudas. Es muy recomendable realizar este seguimiento, pues así se acompaña a los alumnos en el proceso de aprendizaje y mejora el resultado final.

Para empezar, se recomienda que el miembro del equipo que no ve escuche el archivo de audio, anote aquellos sonidos que no entienda y comente cómo se imagina el lugar y el momento en el que transcurre acción, cómo son los personajes y qué están haciendo. De este modo, se inicia un trabajo cooperativo muy interesante, pues los miembros del equipo que sí cuentan con la imagen completan dicha información. Además, como dice el refrán popular "más ven cuatro ojos que dos", así que también es habitual que los miembros "videntes" se fijen en distintos detalles. En conjunto, mediante esta metodología se detectan con más facilidad aquellos aspectos susceptibles de ser audiodescritos. Al final del trabajo, la persona que no había visto el vídeo, tiene acceso a él y, en esa última fase de la revisión, se descubren también aspectos que podrían mejorarse.

Podemos decir que esta actividad colaborativa consigue que el alumnado sea más consciente de las necesidades del público al que se dirige su audiodescripción. Además, al tener que consensuar el producto final entre tres personas, aprenden a escuchar las propuestas de sus compañeros y valorar entre todos cuál es la solución idónea.

Encargos de subtitulación para sordos

En el bloque de subtitulación para sordos realizamos dos encargos simulados por equipos que consistieron en la subtitulación para sordos de fragmentos de cuatro minutos aproximadamente de un cortometraje y de unos dibujos animados, seleccionados cuidadosamente para que contuvieran diferentes tipos de problemas de traducción en los que trabajar. Para empezar a trabajar por equipos y asignar roles a los estudiantes, nos centramos en las diferentes tareas que pueden formar parte de una cadena de trabajo habitual en subtitulación (Sánchez, 2004). Al igual que en el bloque de audiodescripción, trabajamos en equipos de tres estudiantes, uno de los cuales ejercía la función de gestor del proyecto-terminólogodocumentalista, otro la de traductor (encargado de la traducción y el pautado) y otro la de revisor. Además, a este último también se le pedía que simulara ser una persona sorda y viera el producto final, al menos una vez, sin sonido. 
Al igual que en el caso de la audiodescripción, los encargos se empezaban a hacer en el aula y se terminaban fuera de ella. A los alumnos se les proporcionaba el vídeo que debían subtitular con los programas informáticos que habíamos aprendido a utilizar (en esta asignatura hemos trabajado con el uso combinado de los programas de subtitulación gratuitos Subtitle Workshop y Aegisub ${ }^{2}$ ). A los quince días, y tras la tutoría obligatoria (durante la que hablaban con la profesora sobre su proceso de trabajo en equipo y resolvían dudas del encargo), los equipos debían entregar su archivo de subtítulos definitivo y presentar su propuesta de traducción ante el resto de compañeros en la siguiente clase.

Al igual que con las actividades de equipo de audiodescripción, con estas actividades colaborativas de subtitulación para sordos se consigue que el alumnado aprenda a desempeñar diferentes roles dentro de un equipo, a llegar a acuerdos con los compañeros, a tener espíritu crítico y autocrítico, a ponerse en el papel de la persona con problemas de audición, etc. La tutoría obligatoria es también muy importante como forma de seguimiento y guía del trabajo.

\subsubsection{Evaluación por pares}

Otra de las tareas planteadas al alumnado en el módulo de audiodescripción consiste en la evaluación del trabajo de un compañero. Esta actividad está basada en la evaluación por pares. Nuestro propósito es introducirlos en el rigor necesario para acometer el control de calidad de una audiodescripción realizada por otra persona, una de las competencias mencionadas con anterioridad. Esta actividad consta de tres fases:

1. Elaboración y entrega de la audiodescripción de un cortometraje de tres minutos y medio de duración.

2. Revisión de la propuesta de un compañero. Para realizar esta actividad, contaban con el baremo de corrección trabajado en clase y con el que ya habían sido evaluados previamente. Se les indicó que debían marcar no solo los elementos que podrían mejorarse, sino también aquellos aspectos que les parecían especialmente adecuados.

3. Evaluación por parte de la profesora tanto de la audiodescripción como de la corrección.

\footnotetext{
${ }^{2}$ Nos decantamos por el uso de estos programas porque son gratuitos y porque su empleo es relativamente sencillo. El empleo combinado de ambos se debe a que Subtitle Workshop permite a los estudiantes, sobre todo en fases tempranas de aprendizaje, ser mucho más conscientes de las restricciones de caracteres y de velocidad de lectura que Aegisub. Sin embargo, necesitábamos editar posteriormente en Aegisub el archivo de subtítulos creado con Subtitle Workshop para poder añadir colores y colocar subtítulos en diferentes posiciones de la pantalla.
} 
Esta tarea se propone como una de las actividades finales del módulo para comprobar hasta qué punto los alumnos han asimilado las características de una audiodescripción de calidad. Al haber trabajado el fragmento con anterioridad, la fase de revisión les permite descubrir otras opciones y reflexionar sobre su adecuación. Las diferencias existentes entre las distintas audiodescripciones no solo les ayuda a reafirmarse en sus conocimientos, sino que también surgen dudas que son resueltas con posterioridad en la fase de evaluación por parte de la docente, dado que el alumnado recibe en esta actividad una retroalimentación triple. Se evalúa su propuesta de audiodescripción, los comentarios del compañero al respecto y también la revisión que ellos mismos han realizado de otra audiodescripción, ya que tienen acceso a la evaluación de la propuesta que han revisado.

\subsubsection{Taller}

Puesto que el enfoque con el que se plantea la docencia es eminentemente práctico y dirigido al mundo profesional, se invita a la propietaria de una empresa de accesibilidad a impartir un taller de audiodescripción y subtitulación para sordos con la intención de acercar aún más al alumnado a la industria de la accesibilidad. Este taller se realiza una vez finalizado el módulo de audiodescripción y poco antes de que se inicie el de subtitulación para sordos.

En primer lugar, esta profesional cuenta su propia experiencia y elabora un decálogo de los rasgos que caracterizan a un buen audiodescriptor 0 a un buen subtitulador para sordos. Nos parece especialmente importante destacar que este decálogo contiene muchas de las competencias planteadas como objetivos de esta asignatura, de modo que se refuerzan los contenidos trabajados en clase al ser una persona ajena al mundo académico quien confirma la necesidad de adquirir dichos conocimientos.

Tras esta breve introducción, se presenta un ejercicio práctico para cada una de las modalidades. En audiodescripción, se plantea la actividad como una forma de ampliar el trabajo realizado por los alumnos durante el módulo de docencia. Por ello, se propone un ejercicio preparatorio de una audiodescripción en directo. Previamente los alumnos han trabajado las características que distinguen la audiodescripción en directo de teatro y ópera desde un punto de vista teórico. Además, han podido observar el material y el producto final realizado por unos compañeros de una promoción anterior. No obstante, en este taller, tienen la oportunidad de preparar la audiodescripción de un fragmento de la obra teatral La casa de Bernarda Alba representada por la compañía TNT El Vacíe. Una vez 
realizada su propuesta, la comparan con la elaborada por una profesional para la representación de esta obra en el Festival 10 Sentidos. Arte e Integración celebrado en Valencia en noviembre de $2012^{3}$.

Dado que aún no se había iniciado el módulo de subtitulación para sordos, la actividad se concibe como una especie de introducción al proceso de pautado y edición de los subtítulos con especial hincapié en el uso de las tecnologías, ya que pueden ver cómo funciona un programa profesional de subtitulado, del que no se dispone en el aula. Tras mostrar ejemplos de subtitulado para sordos emitidos en televisión y describir los rasgos que los caracterizan, los alumnos subtitulan un fragmento de los dibujos animados Doraemon. Una vez consensuada una única solución entre todo el grupo, los alumnos tienen la posibilidad de ver cómo se editan los subtítulos en el programa profesional de subtitulación.

\subsubsection{Wikis}

Otra de las actividades que realizamos en la asignatura, concretamente dentro del bloque dedicado a la SPS, fue la creación de una wiki colaborativa sobre diferentes aspectos y recursos de SPS. Esta wiki o página web colaborativa se realizó a través del Aula Virtual de la asignatura, y en ella participaron todos los equipos de trabajo. En esta actividad, los estudiantes siguieron trabajando sus competencias interpersonales y recopilaron, por equipos, información que les podía ser útil no solo para la asignatura, sino también para el futuro, en el caso de que decidieran seguir formándose o trabajar en el ámbito de la accesibilidad en los medios audiovisuales. La wiki estaba compuesta por las siguientes siete secciones:

1. Instituciones sobre accesibilidad y asociaciones de sordos

2. Empresas españolas de TAV que ofrecen servicios de SPS

3. Asociaciones profesionales de traductores, y foros y listas de distribución relacionadas con la traducción audiovisual y la SPS

4. Blogs de traductores profesionales y entrevistas relacionadas con la accesibilidad y la SPS

5. Programas informáticos y recursos de ayuda para la traducción para SPS

6. Proyectos de investigación y bibliografía sobre SPS

7. Formación de postgrado en SPS en España y otros países europeos, y charlas o congresos relacionados con la traducción audiovisual y la SPS

\footnotetext{
${ }^{3}$ Más información sobre el festival y la obra de teatro. Disponible en:

http://www.festival10sentidos.com/index.php/qla-casa-de-bernarda-albaq-compania-tnt-el-vacie.html.
} 
Cada equipo de estudiantes se encargó de la edición de una de las siete secciones de la wiki a lo largo del bloque de SPS y, en la última clase, explicó al resto de sus compañeros el contenido de la sección. Cabe decir que resultó sorprendente la variedad y la calidad de los materiales y recursos encontrados, así como el interesante debate generado y el entusiasmo de los estudiantes durante la sesión de presentación de la wiki.

\subsubsection{Portfolio}

La última actividad que realizaron los estudiantes, como paso previo a una prueba final de AD y otra de SPS, fue un portfolio. El portfolio como instrumento didáctico, sin ser ninguna novedad, está muy en boga a día de hoy, especialmente en entornos de aprendizaje cognitivo-constructivistas. Se trata de una carpeta que permite al estudiante presentar actividades, así como reflexiones sobre su proceso de aprendizaje. En cuanto a su formato, aunque en sus orígenes se presentaban como carpetas físicas que contenían documentos en papel, a día de hoy lo más habitual es que sean digitales y que incluyan archivos en diferentes formatos (documentos de texto, vídeos, presentaciones, etc.). En el caso del portfolio de nuestra asignatura, se trató de una carpeta comprimida que cada estudiante debía elaborar individualmente y entregar a través del Aula Virtual el mismo día de la prueba final de AD y de SPS. El contenido del portfolio fue el siguiente:

- La AD y la SPS de equipo corregidas según las revisiones de las profesoras y las aportaciones de los demás estudiantes en clase.

- Una reflexión sobre la experiencia de trabajo colaborativo en los encargos de equipo (organización del trabajo entre los miembros del equipo, características del encargo, problemas y soluciones de traducción, y reflexión sobre el modo de trabajo y el posible beneficio del trabajo en equipo en su proceso de aprendizaje).

- Una reflexión global sobre el aprendizaje a lo largo de la asignatura.

En nuestra opinión, el beneficio de este instrumento es indudable, ya que permitió a los alumnos afianzar las competencias adquiridas a lo largo de la asignatura, reflexionar y autoevaluar su proceso de aprendizaje, y poner de manifiesto aquellos aspectos que más y que menos les habían gustado. Para nosotras, fue también un instrumento muy útil tanto para hacer que los estudiantes consolidaran competencias, como para conocer sus opiniones sobre el trabajo colaborativo y sobre la asignatura y poder pensar en mejoras con vistas a futuros cursos académicos. 


\section{CONCLUSIONES}

Tras la puesta en práctica de todas las actividades mencionadas en este artículo, podemos concluir que se han cubierto sobradamente todos los objetivos propuestos al inicio del curso académico. En primer lugar, los estudiantes han aprendido a resolver problemas de manera autónoma y en equipo. En el portfolio han dejado patente el enriquecimiento que les han proporcionado las actividades de equipo. Asimismo, a lo largo de la asignatura, hemos observado una evolución en la forma de afrontar los problemas que les han surgido.

En segundo lugar, el estudiantado ha aplicado las normas profesionales de la subtitulación para sordos y la audiodescripción para ciegos en España, para lo cual ha necesitado conocer los protocolos de trabajo de la industria de la accesibilidad en España y dominar el manejo de fuentes de documentación sobre lengua, cultura y cine. Tanto el taller como los diferentes encargos de AD y SPS y la evaluación por pares han contribuido al aprendizaje de protocolos profesionales y al desarrollo de su formación como futuros subtituladores y audiodescriptores. Asimismo, la actividad de la wiki colaborativa permitió que pusieran en práctica su dominio de las fuentes de documentación y que indagaran más en diferentes aspectos de accesibilidad audiovisual útiles para el presente y el futuro.

Por último, en cuanto a la valoración global de la asignatura por parte de los alumnos, tanto los cuestionarios de evaluación del taller como las reflexiones individuales expresadas en el portfolio reflejan la buena respuesta del alumnado a la metodología propuesta. Dado el éxito de este enfoque didáctico, proponemos continuar aplicando este tipo de dinámicas en el aula de accesibilidad.

Otra posible actividad que podría introducirse en un futuro en esta misma línea de trabajo colaborativo y enfoque profesional sería la realización de encargos reales de AD y SPS tras establecer, por ejemplo, contacto con clientes de la propia Universidad (otros departamentos, servicio de comunicación, etc.).

REFERENCIAS BIBLIOGRÁFICAS

AENOR, Norma UNE 153020: Audiodescripción para personas con discapacidad visual. Requisitos para la audiodescripción y elaboración de audioguías. Madrid: AENOR, 2005.

AENOR, Norma UNE 153010: Subtitulado para personas sordas y personas con discapacidad auditiva. Madrid: AENOR, 2012. 
Álvarez Doblas, Encarnación; Hidalgo ValdÉs, Miguel, La dimensión práctica en el establecimento de competencias de los subtituladores y audiodescriptores. Sevilla: CEIAF Audiovisual, 2006.

BAdiA, Toni; MatAmala, Anna "La docencia en accesibilidad en los medios". En: TRANS: Revista de Traductología, 2, 2007, pp. 61-71.

BENECKE, Bernd, "Audio-Description". En: Meta: Translators Journal, 49 (1), 2004, pp. 78-80.

CALVO, Elisa; MoRón, Marián, "Propuestas de observación y análisis para la calidad: la traducción e interpretación bajo la lupa". En: $V$ Foro sobre la evaluación de la calidad de la educación superior e investigación. San Sebastián, 2008.

CÁmARA, Lidia; ESPASA, Eva, "The Audio Description of Scientific Multimedia". En: The Translator, 17 (2), 2011, 415-437.

Comisión DEL MERCADO DE LAS TELECOMUNICACIONES, Indicadores de accesibilidad en televisión, 2011. Disponible en: http://informecmt.cmt.es/docs/Anexos/Indicadores\%20accesibilidad\% 20CMT\%202011.pdf [Consultado el 18 de junio de 2013].

DíAZ CINTAS, Jorge, "Por una preparación de calidad en accesibilidad audiovisual". En: TRANS: Revista de Traductología, 2, 2007, pp. 4559.

GonzÁlez DAVIES, Maria, Multiple Voices in the Translation Classroom. Activities, tasks and projects. Amsterdam/Philadelphia: John Benjamins, 2004.

GONZÁLEZ, Julia; WAGENAAR, Robert, Tuning Educational Structures in Europe. Final Report. Phase One. Deusto/Groningen, 2003. Disponible en:

http://www.relint.deusto.es/TUNINGProject/documentos/Tuning_phas e1/Tuning_phase1_full_document.pdf [Consultado el 18 de junio de 2013].

HUERTAS BARROS, Elsa, "Collaborative learning in the translation classroom: preliminary survey results". En: Jostrans: the Journal of Specialised Translation, 16, 2011. Disponible en: http://www.jostrans.org/issue16/art_barros.php. [Consultado el 18 de junio de 2013].

ITC, Guidance on Standards for Audio Description, 2000. Disponible en: www.ofcom.org.uk/static/archive/itc/uploads/ITC_Guidance_On_Stan dards_for_Audio_Description.doc [Consultado el: 18 de junio de 2013].

JAKOBSON, Roman, "On linguistic aspects of translation". En: Lawrence Venuti (ed.): The Translation Studies Reader. London y New York: Routledge, 1959 (2000), pp. 113-118. 
KELLY, Dorothy, "La competencia traductora: bases para el diseño curricular". En: Puentes, 1, 2002, pp. 9-20.

, A Handbook for Translator Trainers. A Guide to Reflective Practice. Manchester: St. Jerome, 2005.

KIRALY, Donald, A Social Constructivist Approach to Translator Education. Empowerment from Theory to Practice. Manchester: St. Jerome, 2000.

MARZÀ IBAÑEZ, Anna, "Evaluation criteria and film narrative. A frame to teaching relevance in audio description". En: Perspectives, 18(3), 2010, pp. 143-153.

Matamala, Anna; Orero, Pilar, "L'accessibilitat a Televisió de Catalunya: parlem amb Rosa Vallverdú, directora del departament de Subtitulació de TVC". En: Quaderns: Revista de traducció, 16, 2008, pp. 301-312.

MuÑoz RaYA, Eva (coord.), Libro Blanco. Título de Grado en Traducción e Interpretación. Agencia Nacional de Evaluación de la Calidad y Acreditación, 2004. Disponible en: http://www.aneca.es/media/150288/libroblanco_traduc_def.pdf [Consultado el 18 de junio de 2013].

NEVES, Josélia, Guia de Legendagem para Surdos. Vozes que se vêem. Leiria y Aveiro: Instituto Politécnico de Leiria y Universidade de Aveiro, 2007.

, "Training in subtitling for the d/Deaf and the hard-of-hearing". En: Jorge Díaz-Cintas (ed.), The Didactics of Audiovisual Translation. Amsterdam y Philadelphia: John Benjamins, 2008, pp. 171-189.

PACTE, "La Competencia traductora y su adquisición". En: Quaderns: Revista de Traducció, 6, 2001. Disponible en:

http://grupsderecerca.uab.cat/pacte/sites/grupsderecerca.uab.cat.pact e/files/2001b_PACTE_QUADERNS.pdf. [Consultado el 18 de junio de 2013].

PACTE, "Building a Translation Competence Model". En: Fabio Alves (ed.), Triangulating Translation: Perspectives in Process Oriented Research. Amsterdam: John Benjamins, 2003. Disponible en: http://grupsderecerca.uab.cat/pacte/sites/grupsderecerca.uab.cat.pact e/files/2003_PACTE_Benjamins_0.pdf. [Consultado el 18 de junio de 2013].

PALOMO, Alicia, Estudio descriptivo y comparativo de la audiosdescripción para niños con deficiencias auditivas en España y Gran Bretaña. Trabajo de investigación inédito. Castellón de la Plana: Universitat Jaume I, 2009.

PYM, Anthony, "A definition of translational competence applied to the teaching of translation". En: Mladen Jovanovic (ed.), Translation: A 
Creative Profession: $12^{\text {th }}$ World Congress of FIT, Prevodilac, Belgrado, 2001, pp. 541-546.

Royal National Institute Of Blind People, Audio Description for Children, 2009. Disponible en:

http://www.rnib.org.uk/professionals/documents/ad_for_children_guid elines.doc [Consultado el 18 de junio de 2013].

SÁNCHEZ, Diana, "Subtitling methods and team-translation". En: Pilar Orero (ed.), Topics in Audiovisual Translation. Amsterdam y Philadelphia: John Benjamins, 2004, pp. 9-17.

SNYDER, Joel, "Audiodescription: the visual made verbal". En: Jorge DíazCintas (ed.), The Didactics of Audiovisual Translation. Amsterdam y Philadelphia: John Benjamins, 2008, pp. 191-198. 